\title{
Ranked Sparsity: A Cogent Regularization Framework for Selecting and Estimating Feature Interactions and Polynomials
}

Ryan A. Peterson, Joseph E. Cavanaugh

\begin{abstract}
We explore and illustrate the concept of ranked sparsity, a phenomenon that often occurs naturally in modeling applications when an expected disparity exists in the quality of information between different feature sets. Its presence can cause traditional and modern model selection methods to fail because such procedures commonly presume that each potential parameter is equally worthy of entering into the final model - we call this presumption "covariate equipoise". However, this presumption does not always hold, especially in the presence of derived variables. For instance, when all possible interactions are considered as candidate predictors, the premise of covariate equipoise will often produce over-specified and opaque models. The sheer number of additional candidate variables grossly inflates the number of false discoveries in the interactions, resulting in unnecessarily complex and difficult-to-interpret models with many (truly spurious) interactions. We suggest a modeling strategy that requires a stronger level of evidence in order to allow certain variables (e.g. interactions) to be selected in the final model. This ranked sparsity paradigm can be implemented with the sparsity-ranked lasso (SRL). We compare the performance of SRL relative to competing methods in a series of simulation studies, showing that the SRL is a very attractive method because it is fast, accurate, and produces more transparent models (with fewer false interactions). We illustrate its utility in an application to predict the survival of lung cancer patients using a set of gene expression measurements and clinical covariates, searching in particular for gene-environment interactions.
\end{abstract}

Keywords: derived variables, feature selection, information, lasso, model selection

Declarations: Not applicable.

Date last modified: $12 / 06 / 2021$

Availability of data and material: Data used in this application is publicly available via GEO database accession number GSE68465.

Code availability: Code for simulations and methods is included as supplemental material.

\author{
Ryan Peterson \\ Department of Biostatistics \& Informatics, University of Colorado School of Public Health, Aurora, CO \\ email: ryan.a.peterson@cuanschutz.edu (corresponding author) \\ Joseph Cavanaugh \\ Department of Biostatistics, University of Iowa College of Public Health, Iowa City, IA \\ email: joe-cavanaugh@uiowa.edu
}




\section{Introduction}

In the ever-growing, ever-changing field of model selection and machine learning, "black-box" predictive models (e.g. neural networks) have become increasingly popular (and increasingly opaque). When one's exclusive desire is predictive accuracy, these difficult-to-interpret models are often worth a certain lack of understanding. However, overly complex predictive contexts are not generally compatible with the traditional aim of science: to explain and to understand the world in which we live. With their growing popularity, black-box models are starting to be applied in situations where explanation should be the primary goal. Worse yet, in some circumstances, there is little regard for the consideration that more transparent models could produce similar prediction results. So, as scientists continually increase the number of candidate predictors, those building models are using increasingly complicated functions of candidate predictors in order to optimize for predictive performance above all else. Is there a justifiable way to hold on to the traditional aims of science amid these trends?

In this paper, we will argue that the benefits reaped from choosing a black-box model must be weighed against the interpretative costs of a lack of scientific understanding. However, before we can proffer a method to accomplish this goal, we must first answer a salient question - why do black-box methods outperform transparent models in prediction? The answer is difficult because these black-box methods are diverse, as are the situational considerations that make a particular method perform better or worse. Broadly speaking, the benefits of black-box methods can be roughly explored by investigating situations where transparent linear models fail. We will focus in particular on the issue of bias caused by model misspecification.

Say we have a vector of data made up of a response of interest $\boldsymbol{y}$ and a matrix of covariates (or predictors) $X$, some columns of which are related to $\boldsymbol{y}$ while others are not. Suppose that the variates comprising $\boldsymbol{y}$ are independent (conditional on $X$ ) and can be conceptualized as following a distribution in the exponential family. One can envision many ways of fitting an optimal predictive model to $\boldsymbol{y}$, but a popular method (if transparency is a goal) is to fit generalized linear models (GLMs) based on all possible subsets of covariates to select the best model on the basis of an information criterion. This method is somewhat limited to lower-dimensional settings, because the number of candidate models increases combinatorically with the dimension of $X$. However, in recent times the Least Absolute Shrinkage and Selection Operator (the lasso) has changed the landscape surrounding the problem of identifying a suitable predictive model (Tibshirani, 1996). With the lasso and its many extensions, it is possible to have an extremely high-dimensional covariate space and still end up with a relatively well-fit, interpretable model. In either setting, if the true generating model has informative interactions among covariates and/or meaningful nonlinear covariate-response relationships, black-box methods exist that can outperform even the best traditional or lasso model (given enough data). 
This is because none of the candidate main effect (i.e. transparent) models can capture the model's complex interaction/polynomial terms; all of the candidate transparent models are misspecified.

One potential solution, given the power and flexibility of the lasso, would be simply to add "derived variables" of $X$, such as interactions and polynomials, into a new (potentially very large) design matrix. The lasso can simultaneously select and estimate important interactions and polynomials even in this ultra-high dimensional setting. However, in this paper, we will show that this method yields a preponderance of both false and missed discoveries, unless proper methods are used to incorporate what we call "ranked sparsity."

The paper is organized as follows. First, we intuitively motivate the concept of ranked sparsity, illustrating its necessity when looking for active interactions. Second, we propose the sparsity-ranked lasso, and connect it to some other related concepts and regularization methods that have been proposed in the literature, as well as other state-of-the-art interaction selection methods. Next, we present simulation studies to investigate the performance of the SRL compared to competing methods in the polynomial and interaction selection setting. We then apply the SRL in a high-dimensional setting of gene-environment interaction selection in the context of a lung cancer application. Finally, we discuss the strengths and weaknesses of the SRL relative to other strategies that have been proposed.

\section{Ranked Sparsity}

\subsection{Intuitive Motivation}

Ranked sparsity, which we also refer to as ranked skepticism, is a philosophical framework that challenges the traditional implementation of Occam's Razor in the context of variable selection. In Einstein's words ${ }^{1}$, the maxim stipulates that "everything must be made as simple as possible, but not simpler." This is a noble goal, but some obvious questions arise: how do we know when a model is as simple as it should be? How should we measure simplicity in the first place? Specifically, we wish to challenge the ubiquitous answers to these questions in the field of model selection, which rely on a presumption that we call "covariate equipoise": the prior belief that all covariates are equally likely to enter into a model. To illustrate this idea, say we are trying to find a well-fit model to predict an outcome $\boldsymbol{y}$ using a set of covariates, including age, weight, and height. Of the candidate models below, which is "simpler"?

$$
\begin{aligned}
& E\left(y_{i}\right)=\beta_{0}+\beta_{1} \text { Age }_{i}+\beta_{2} \text { Weight }_{i}+\beta_{3} \text { Age }_{i} * \text { Weight }_{i} \\
& E\left(y_{i}\right)=\beta_{0}+\beta_{1} \text { Age }_{i}+\beta_{2} \text { Weight }_{i}+\beta_{3} \text { Height }_{i}
\end{aligned}
$$

\footnotetext{
${ }^{1}$ Debate exists regarding whether this is a true quote or a paraphrase of Einstein.
} 
Virtually all variable selection tools assume these two models to be equally simple. Due to the presumption of covariate equipoise, simplicity is equated to parsimony, and is measured only by the number of parameters in the model (which is 4 in both models). However, any statistician would quickly recognize that model (2) is an order of magnitude easier to understand and communicate than model (1). We argue that a proposed model's simplicity should therefore not only be tied to its level of parsimony, but also to its transparency as measured by the ease at which it can be understood and communicated (a metric loosely tied to the number of interactions and nonlinear terms in the model). Ultimately, a good model interpreted correctly is better than a great model interpreted erroneously. This concept is the primary motivation for the ranked sparsity methods we introduce in this paper, which provide a means of searching for important interactions/nonlinear terms without rendering the chosen model unnecessarily opaque.

In this work, for the linear form used to characterize the mean outcome, we use the term "main effects" to refer to the regression coefficients on the original covariates of interest, and "interaction effects" to refer to coefficients on the product of covariates that correspond to the main effects. We also define the "sparsity level" as the proportion of candidate variables (a.k.a. features, covariates, or predictors) that are inactive in a given true generating model. A high sparsity level thus indicates that a smaller proportion of candidate variables are truly important. Conversely, the "saturation level" is defined as the proportion of candidate variables that are active. The sparsity level is governed by a mix of what cannot be known about nature's true generating model and what can (sometimes) be known about the ambition of a particular scientific project. In sparse settings, consistent model selection criteria such as the Bayesian Information Criterion (BIC) (Schwarz, 1978) and its extensions (Bogdan et al., 2008; Chen and Chen, 2008) have been shown to be effective, while in saturated settings, efficient criteria such as $\mathrm{Cp}$, AIC, and corrected AIC (Mallows, 1973; Akaike, 1974; Hurvich and Tsai, 1989) perform relatively well. This difference in the performance of various model selection criteria suggests that in settings where multiple levels of sparsity are to be expected among different groups of covariates, the optimal criterion needs to account for this disparity in some way by penalizing the covariates differently. The "ranking" that occurs in our concept of ranked sparsity thus refers to settings where the sparsity levels within covariate groups are expected to be ordered in a specific way $a$ priori.

With this impetus in mind, given a saturation level in the main effects, we can show that the maximum saturation level attainable for the (first-order) interaction effects is limited by "hierarchy" assumptions about the true generating model. Sometimes called model heredity or the marginality principle, model hierarchy refers to the rules pertaining to which interactions can be nonzero, and it is typically broken down into "strong," e.g. $E\left(y_{i}\right)=\beta_{0}+\beta_{1} x_{1 i}+\beta_{2} x_{2 i}+\beta_{3} x_{1 i} * x_{2 i}$; "weak," e.g. $E\left(y_{i}\right)=\beta_{0}+\beta_{1} x_{1 i}+\beta_{3} x_{1 i} * x_{2 i}$; and "anti-" (or "non-") hierarchical models, e.g. $E\left(y_{i}\right)=\beta_{0}+\beta_{3} x_{1 i} * x_{2 i}$. As an illustration of how hierarchy 
limits saturation, consider a case where only 3 of 30 possible main effects are active, then strong hierarchy would dictate that in the generating model, only $\left(\begin{array}{l}3 \\ 2\end{array}\right)=3$ signal variables can exist in the interaction set. Under weak hierarchy, then this quantity is limited to $\sum_{j=1}^{3}(30-j)=84$ active interactions. In either case, the number of signals in the interaction set is bounded by the number of signal variables in the main effects, as are their saturation (sparsity) levels (see supplemental materials for proofs). Of course, outside of simulation settings, the hierarchy status of the generating model will be unknown. Even so, there is good reason to believe that the saturation level in one set of covariates (interaction effects) is going to be less than the saturation level in another group of covariates (main effects). As a result, it becomes necessary to account for this disparity somehow in the model selection process; we cannot simply apply the same penalty to both main effects and interactions and expect optimal performance.

\subsection{The Sparsity-Ranked Lasso}

In this section, we propose and motivate the Sparsity-Ranked Lasso (SRL) as a tool for implementing ranked sparsity in the search for important derived variables of a feature space. Suppose we have $p$ features $\left[x_{1}, x_{2}, \ldots, x_{p}\right]=X_{n \times p}$, and a centered response variable $\boldsymbol{y}$; some (but not all) features are related to $\boldsymbol{y}$. For this section, we assume that the variates comprising $\boldsymbol{y}$ are normally distributed and conditionally independent given $X$; however, it will become clear that the concept and development apply more generally in the GLM family. The lasso (Tibshirani, 1996) has become immensely popular in this setting for its computational efficiency and its effectiveness in variable selection. The lasso simultaneously estimates coefficients for each of

the $p$ features and selects from them, such that they are either "active" (i.e. $\left.\hat{\beta}_{j} \neq 0\right)$, or "inactive" $\left(\hat{\beta}_{j}=0\right)$. The estimated nonzero coefficients do suffer from a bias that is introduced by the lasso's penalty term, but this bias is often warranted as it significantly attenuates the variance associated with having too saturated of a model. Typically, the magnitude of shrinkage induced by the lasso's penalty term is treated as a tuning parameter $(\lambda)$ and selected on the basis of an information criterion or cross-validation $(\mathrm{CV})$. In this section, we will show how the lasso is expected to fail when applied to feature sets of different sizes, most notably when applied to interactions and main effects, and we will offer a solution via the sparsity-ranked lasso.

The ordinary lasso solution can be obtained by standardizing all of the columns of $X$ and minimizing the following expression with respect to $\beta$ :

$$
\|\boldsymbol{y}-X \boldsymbol{\beta}\|^{2}+\lambda \sum_{j=1}^{p}\left|\beta_{j}\right|
$$

It is well-known that this solution has a Bayesian interpretation. If each $\beta_{j} \sim \operatorname{Laplace}(0, \lambda)$, then the mode of the joint posterior distribution represents the lasso solution (Tibshirani, 1996) for a given $\lambda$ value. As 
the sample size increases, the likelihood becomes more concentrated and contributes more information to the posterior, eventually pulling the mode off of zero. As $\lambda$ is increased, the balance of information shifts toward the Laplace prior, and the mode gets pulled (potentially all the way) to zero (a visualization of this is available at https://ph-shiny.iowa.uiowa.edu/rpterson/shiny_vis1/). These zero-centered independent Laplace priors form the following joint prior density:

$$
\pi(\boldsymbol{\beta})=\prod_{j=1}^{p} \frac{\lambda}{2} e^{-\lambda\left|\beta_{j}\right|}
$$

As a brief aside, we turn to the concept of Fisher information. Fisher information is invoked in likelihood theory to describe the behavior of maximum likelihood estimators, but the concept can quantify the structural characteristics of any joint density. ${ }^{2}$ For $W \sim f(w \mid \lambda)$ where $\lambda \in \Lambda$ is scalar and $\lambda \rightarrow \log f(w \mid \lambda)$ is twice differentiable in $\lambda$ for every $w$, the model Fisher information at any $\lambda$ is defined to be $I(\lambda)=$ $E_{W \mid \lambda}\left[-\frac{\partial^{2}}{\partial \lambda^{2}} \log f(W \mid \lambda)\right]$.

Now, consider partitioning the covariate space $X$ into $K$ groups, such that $X=\left[A_{1}, A_{2}, \ldots, A_{k}, \ldots, A_{K}\right]$. If we let $p_{k}$ refer to the column dimension of $A_{k} \forall k$, and let $\beta_{j}^{k}$ refer to a particular $\beta_{j}$ in covariate group $k$, then the prior for $\beta$ undergoes a purely cosmetic change and becomes

$$
\pi(\boldsymbol{\beta} \mid \lambda) \propto \prod_{k=1}^{K} \prod_{j=1}^{p_{k}} \lambda e^{-\lambda\left|\beta_{j}^{k}\right|}
$$

If we think of all of the $\beta_{j}^{k}$ as random variables (which they are a priori) and take $\lambda$ to be a parameter, it becomes straightforward to find the Fisher information in this prior density.

$$
\begin{array}{r}
\frac{\partial^{2}}{\partial \lambda^{2}} \log \pi(\boldsymbol{\beta} \mid \lambda)=-\frac{1}{\lambda^{2}} \sum_{k=1}^{K} p_{k} \\
I(\lambda)=E_{X \mid \lambda}\left[-\frac{\partial^{2}}{\partial \lambda^{2}} \log \pi(\boldsymbol{\beta} \mid \lambda)\right]=\frac{1}{\lambda^{2}} \sum_{k=1}^{K} p_{k}
\end{array}
$$

This information increases with the dimension of each group's parameter space equally (for any $\lambda>0$ ). So if $p_{1}=10$, and $p_{2}=100$, by default the contribution toward the prior information by the covariate group $A_{1}$ is only one tenth that of the covariate group $A_{2}$, for any $\lambda>0$. If $A_{1}$ refers to the main effects, and $A_{2}$ refers to their pairwise interactions, there can be a substantial degree of a priori informational asymmetry between the interactions and the main effects. This asymmetry leads popular feature selection tools such as the "all-pairwise lasso" (APL) to select too many selections among the candidate interaction effects while shrinking the main effects excessively.

In many (perhaps most) situations, the preceding weighting scheme may not be desired. We can slightly

\footnotetext{
${ }^{2}$ Jeffreys, for instance, derived his famous noninformative prior based on the concept of the Fisher information of a prior density.
} 
modify the prior distribution by replacing $\lambda$ with $\lambda_{k}=\lambda \sqrt{p_{k}}$. Now, unlike before when the distributions were independent and identical for all $k$, each $\beta_{j}^{k}$ is only independent and identically distributed within its own covariate group $k$. The Fisher information contained in the prior for covariates in group $k$ after this modification is

$$
I\left(\lambda_{k}\right)=\frac{p_{k}}{\lambda_{k}^{2}}=\frac{p_{k}}{\lambda^{2} p_{k}}=\frac{1}{\lambda^{2}} \forall k
$$

In words, by scaling each group's penalty by the square-root of its dimension, we have ensured that the prior information is the same across groups; no group has an a priori informational advantage. Therefore, we can achieve a "ranking" in the sparsity that treats covariate groups equally as opposed to the covariates themselves. If we add another tuning parameter, $\gamma$, in the definition for $\lambda_{k}$ such that $\lambda_{k}=\lambda p_{k}^{\gamma}$, the resulting approach can be seen as a generalization the ordinary lasso, where

$$
I\left(\lambda_{k}\right)=\frac{1}{\lambda^{2}} p_{k}^{(1-2 \gamma)}
$$

If $\gamma=0$, this is identical to the ordinary lasso. If $\gamma=0.5$, then $w_{k}=\sqrt{p_{k}}$ and each covariate group contributes the same amount of prior information (which is a good default setting for many circumstances, especially for the context of interactions). As $\gamma$ increases, the penalties for larger groups of covariates increase quickly (as the information contribution decreases quickly with group size). In less-common cases where the grouping is not well-defined, we suggest tuning $\gamma$ to a value between zero (the ordinary lasso) and 0.5 (equal group-level prior information). Under this primary SRL formulation, we do not suggest choosing $\gamma>0.5$ unless there is a strong reason to believe that the quality of information decreases substantially with group size.

We consider a variant of the penalty weighting scheme where the information is expected to decrease as the group index increases. Specifically, instead of weighting the penalties by $p_{k}^{1-2 \gamma}$, we use $\left(\sum_{i=1}^{k} p_{i}\right)^{1-2 \gamma}$. This group index formulation yields a group-level information contribution of

$$
I\left(\lambda_{k}\right)=\frac{1}{\lambda^{2}}\left(\frac{p_{k}}{\sum_{i=1}^{k} p_{i}}\right)^{(1-2 \gamma)}
$$

In words, the information contribution is highest for $A_{1}$, and decreases cumulatively as more groups are added. If $A_{1}$ represents main effects, and $A_{2}$ represents squared polynomial terms of those main effects, this cumulative group index penalty ensures that the polynomial terms are penalized more heavily than the main effects (despite having the same group size). We call this formulation the cumulative SRL, for which the value of $\gamma$ determines the extent to which penalty increases with the group index (e.g. polynomial order). We suggest using cross-validation to tune $\gamma$ for the cumulative SRL.

With these changes, the objective function resembles closely that for the adaptive lasso (Zou, 2006), 
minimizing the following with respect to $\beta$ :

$$
\|\boldsymbol{y}-X \boldsymbol{\beta}\|^{2}+\lambda \sum_{k=1}^{K} \sum_{j=1}^{p_{k}} w_{k}\left|\beta_{j}^{k}\right|
$$

Unlike the adaptive lasso, $w_{k}$ only depends on the group dimensions and potentially $\gamma$ and the group index.

Specifically, $w_{k}=p_{k}^{(1-2 \gamma)}$ for the original SRL $\left(w_{k}=\sqrt{p_{k}}\right.$ assuming group parity in prior information), and $w_{k}=\left(\frac{p_{k}}{\sum_{i=1}^{k} p_{i}}\right)^{(1-2 \gamma)}$ for the cumulative SRL. As has been shown in other work (e.g. Wang and Wang, 2014), this objective function can be slightly modified to handle non-normal outcomes (binary, Poisson, survival, etc.) by substituting the negative log-likelihood for the least squares term, minimizing $-l(\boldsymbol{\beta})+\lambda \sum_{k=1}^{K} \sum_{j=1}^{p_{k}} w_{k}\left|\beta_{j}^{k}\right|$ with respect to $\beta$; such a substitution was employed for this paper's application. We suggest using path-wise coordinate descent to optimize this objective function with respect to $\boldsymbol{\beta}$; this can be implemented with either the sparseR package or ncvreg (Breheny and Huang, 2011).

To summarize, while the ordinary lasso presumes throughout its path that the sparsity levels are equal among covariate groups, the SRL can enforce a ranking in the expected sparsity levels such that the amount of contributed prior information is controlled across covariate groups. We also introduced a "cumulative" variant of the SRL, which is particularly useful for selecting and estimating polynomial effects. We illustrated how either the primary SRL or its cumulative variant can be tuned with $\gamma$. A sensible choice for the SRL is to utilize $\gamma=0.5$, which sets the prior information to be equal across all covariate groups; we suggest fixing $\gamma=0.5$ when utilizing the SRL specifically for interactions or for otherwise well-defined covariate groups (or both, as is the case in this paper's application). The cumulative SRL performs best when $\gamma$ is tuned among several possible values (e.g. $\gamma \in\{0, .5,1,2,4,8, \ldots\}$, suggesting an increased amount of penalization for higher orders of polynomials), which can be done using an information criterion or CV. We explore the performance of the SRL in the forthcoming simulation studies and application, after a brief discussion of similar ideas and techniques in the literature.

\subsection{Related Methods}

\subsubsection{Existing Methods which Penalize Based on Group}

Several methods exist that are close in spirit to a general ranked sparsity framework, including the Integrative Lasso with Penalty Factors (IPF-lasso) (Boulesteix et al., 2017) and the priority lasso (Klau et al., 2018). In both methods, each group of covariates has its own estimated penalty. The IPF-lasso creates a new tuning parameter for each covariate group $k$, estimating $\lambda_{k}$ using a grid search and cross-validation. While technically possible for any number of groups, the IPF-lasso can become computationally difficult when multiple groups are considered (though notably, an adaptive extension of the IPF-lasso which mitigates 
this issue has been proposed and implemented in the ipflasso R package). Similarly, the priority lasso incorporates a priority ordering of feature groups and fits sequential lasso models on these groups, using residuals from each model as a new outcome to predict using next most important feature set. The priority lasso is feasible for multiple groups; however in order to avoid over-optimism, Klau et al. (2018) recommend a cross-validated offset schema which can be computationally intensive and difficult to implement for multiple groups.

Another related lasso extension meriting discussion is the sparse group lasso (SGL). The solution to the SGL is found by minimizing the following with respect to $\beta$ :

$$
\|\boldsymbol{y}-X \boldsymbol{\beta}\|^{2}+\alpha \lambda \sum_{k=1}^{K} \sum_{j=1}^{p_{k}}\left|\beta_{j}^{k}\right|+(1-\alpha) \lambda \sum_{k=1}^{K} \sqrt{p_{k}} \| \boldsymbol{\beta}^{k}||
$$

SGL bears a resemblance to the SRL, noticing in particular the factor multiple of the group-level penalty, $\sqrt{p_{k}}$. However, despite the similar penalty scaling, the SGL will yield quite different results to the SRL in practice. While SGL shrinks the magnitude of the entire vector $\boldsymbol{\beta}^{k}$ within each group, SRL penalizes each coefficient in some sense independently from the others in its group. For example, if the magnitude of the first coefficient in group one is large, i.e. $\hat{\beta}_{1}^{1}>>0$, the SRL would not induce any effect on the magnitude of $\hat{\beta}_{2}^{1}$. The SGL still penalizes each variable separately in its first penalty, but its second penalty is on the group-level magnitude. In our hypothetical example, a large $\hat{\beta}_{1}^{1}$ coefficient would thus relax the penalty on $\hat{\beta}_{2}^{1}$ to an extent. See Figure 1 in Friedman, Hastie and Tibshirani (2010) for a good visualization of this principle. A more detailed comparison of the performance of the SGL to the SRL is left for future work, but one benefit of the SRL that is already evident is its lack of the need for an additional tuning parameter, as $\gamma=0.5$ assumes equal prior information across groups, and is thus a defensible choice for many circumstances.

\subsubsection{Existing Methods for Interaction Selection}

Several methods have been proposed for selecting and estimating interactions under the weak and/or strong hierarchy "constraint." The hierNet approach (Bien, Taylor and Tibshirani, 2013) is well-suited for low-dimensional problems due to its computational complexity. A similar regularization-based method, glinternet (Lim and Hastie, 2015), has been shown to be as effective as hierNet in selecting interactions, but able to execute the fitting and selection 10-10000 times faster. The "strong heredity interaction model" (SHIM) approach is similar to the hierNet approach; it extends the lasso to select interaction terms while under a strong hierarchy constraint. SHIM also adds an adaptive lasso element to achieve the oracle property (Choi, $\mathrm{Li}$ and $\mathrm{Zhu}, 2010$ ), and uses an IPF-lasso-type approach of tuning the penalty for the interactions separately from the main effects. SHIM thus has an additional tuning parameter to cross-validate over. Yet another 
approach is called "regularization under marginality principle" (RAMP) (Hao, Feng and Zhang, 2018), which is a two-stage regularization approach that is useful for settings where the storage of the interaction model matrix is an issue. By having a first-stage screening via regularization on the main effects, RAMP substantially cuts down on the size of the model matrix in its second stage, only considering candidate interactions that made it past the first selection stage. All of these methods constrain the solution path to weakly or strongly hierarchical models. Another set of 12 alternative methods for determining treatment-biomarker interaction screening via various types of regularization and dimension reduction are described and empirically evaluated in Ternès et al. (2017).

It is important to note that many of these methodological works on interaction selection involve a comparison to the APL, which consistently selects too many interactions in these comparisons. This issue gets compounded when the true generating model has very few "active" interactions, which is an ongoing limitation of interaction feature selection for some of these methods (Lim and Hastie, 2015). The SRL, we will show, does not suffer this limitation. Further, one consideration that is often mentioned only briefly in these related works is whether or not we should restrict all candidate models to be hierarchical. The usual presumption is that it makes the most sense for all candidate models to be hierarchical. However, Chipman (1996) provides a compelling paradigm for model hierarchy in a Bayesian context, and argues why the strict imposition of hierarchical structures may not always be defensible. If we think of interactions as children of their "parent" main effects, we would guess that a child is certainly most likely to be in a model if its parents are both in the model. It is comparably less probable that a child is in a model if one of its parents is not. Is it absolutely impossible (with probability zero) for a child to be in a model without either of its parents?

There are numerous occasions where a generating model is not, in fact, hierarchical. Chipman gives the example of the atmospheric sciences, where relations of the form $Y=A \exp (B C)$ are common, which is a non-hierarchical model on the log scale. We can also point to models for lung cancer, where "pack-years," the interaction between the number of years spent smoking and the reported number of cigarette packs smoked per day, is an acknowledged risk factor on its own. In fact, a non-hierarchical model of this type is plausible in any setting where level of exposure and time of exposure are both captured somewhere in the candidate covariate space. Therefore, we argue that in lieu of hierarchy constraints, a better general rule would be to enforce hierarchy preference. This is considerably different than a constraint. We have shown that the SRL enforces higher penalties for interactions than for the main effects (when $p>2$ ), which naturally enables hierarchy preference (but does not force hierarchy).

Finally, it is feasible that the IPF-lasso could be used to select from all possible interactions by defining main effects and interaction effects as two separate blocks, an approach which would enforce neither a hierarchy preference nor a constraint. Such an approach, while defensible, may suffer from imprecision (in 
ensuring that interactions and main effects contribute proportionally to the prior information). Boulesteix et al. (2017) advise to investigate penalty factors within each group as $\left(1,2^{\gamma}\right)$ for a sequence of positive and negative integers $\gamma$ (in the two-group case). For interactions, if candidate $2^{\gamma}$ values are not near $\sqrt{\left(\begin{array}{l}p \\ 2\end{array}\right)}$, we would expect asymmetry in the prior information. More generally, similar imprecision is likely to occur when covariate groups vary substantially in size. For example, in one application, Boulesteix et al. (2017) investigate combining clinical data (11 features) with microarray gene expression measurements $(22,283$ features) to predict the survival of patients with breast cancer. Their CV procedure estimated the optimal penalty factor for the genetic expression data to be $2^{5}=32$, thereby penalizing the expressions much more than the clinical data (in fact, there were no selected gene expressions). With the SRL, prior to CV, we know that for the gene-expression measurements to contribute the same amount of prior information as the clinical features, this penalty factor should be $\sqrt{22,283 / 11} \approx 45$. Therefore, despite being optimized via CV, 32 is likely too low for the genetic features; a penalty factor of 45 would still select no expression features, in that sense the model would be the same. However, with a penalty factor of only 32 , the coefficients on the clinical features may be shrunk more than necessary, thereby decreasing the power to detect clinical effects as well as (perhaps) the predictive accuracy of the final model.

\subsection{The sparseR Package}

We have developed an R package, sparseR, which works in concert with ncvreg (Breheny and Huang, 2011) to implement and facilitate the ranked sparsity methods discussed in this paper. By building upon the recipes package (Kuhn and Wickham, 2019), sparseR also provides a useful means of preprocessing data sets before model fitting, which can facilitate the use of a mix of factors, binary variables, and continuous variables as covariates. The package also contains an information-criterion based metric that we call RBIC (Peterson, 2019), which is paired with a forward step-wise selection function that can select from all possible interactions and polynomials under strong, weak, or non-hierarchy using the ranked-sparsity framework. The sparseR package and a detailed tutorial will soon be made available on the Comprehensive R Archive Network (CRAN). A development version is available on GitHub at https://github.com/petersonR/sparseR.

\section{Simulations}

\subsection{Polynomial Simulation Study}

Consider a simple simulated example, where we have 100 observations arising from a true $f(x)=10 *(x-.5)^{2}$ measured with some residual noise, $\varepsilon_{i} \stackrel{i i d}{\sim} N\left(0,0.9^{2}\right)$, and $x_{i} \stackrel{i i d}{\sim}$ unif $(0,1)$. This relationship is shown by the 
solid black line in Figure $1^{3}$. It is well-known that the addition of extraneous polynomial terms in a regression model hurts the model's predictive performance, especially at the bounds of the covariate space. This can be seen in the top three plots of Figure 1 - adding higher order terms increases the "wiggliness" of the fits (represented by the grey lines).

With only one covariate, we could easily fit, say, $m$ models with increasing orders of polynomials up to $m$ and select the best order using an information criterion. However, in higher dimensional settings with $p$ covariates, this approach is not practical since the number of possible models is $2^{p m}$. In such settings, one might think to use the lasso to select the optimal order - this method is explored in the middle 3 plots of Figure 1. Evidently, the bias incurred by the L1 penalty reduces some of the variability (the "wiggliness") in the relationship, while at the same time contaminating the shape of relationship (note that the fitted lines are bent down towards the origin).

The cumulative SRL can be successfully applied for such polynomial models; if we set $w_{j}=\left(d_{j}\right)^{\gamma}$, where $d_{j}$ refers to the degree of covariate $j$, this approach is equivalent to the cumulative group-index SRL in the one-covariate case. The resulting fits, where $\gamma \in\{0.5,1,2\}$ is selected by $\mathrm{BIC}^{4}$, are shown by the grey lines in the bottom three plots of Figure 1. We observe that the fits are both reducing the wiggliness from the extraneous terms while at the same time inducing less "bending" towards the origin.

The plots in Figure 1 only show 50 fits each, but repeating this process 10,000 times, we can compare the root-mean-squared error (RMSE) of estimation resulting from each method across the domain of $x^{5}$. In Figure 2, we show the increase in the RMSE for each method relative to a baseline "oracle" model (i.e. an ordinary least-squares model that only includes the "true" $x$ and $x^{2}$ ). We find that while there is no replacement for an "oracle" model, the next best models are those which utilize the SRL method. Interestingly, there appears to be very little in terms of predictive difference between the SRL applied up to the 4th order and SRL applied up to the 6th order; this implies that we could likely increase the maximum order and still not observe a substantive impact on the predictive performance. On the other hand, if the lasso is used, there is a marked decrease in predictive performance between the 4 th order model and the 6 th order model; it appears as though these models (as well as the OLS models) perform increasingly poorly as the number of extraneous polynomials increases.

We conducted a separate simulation study to further establish the performance of the cumulative SRL for more general functional forms. Between kernel and spline methods, many options exist for estimating a smooth relationship in low-dimensional settings. We investigate four possible generating models: polynomials

\footnotetext{
${ }^{3}$ The $\mathrm{R}$ language and environment version 4.0.2 is used for all figures in this work.

${ }^{4}$ We exclude $\gamma=0$ in this application of the cumulative SRL to showcase how the method differs from the ordinary lasso applied to the polynomials, which is equivalent to setting $\gamma=0$.

${ }^{5}$ The RMSE of estimation is based on the sum of the squared deviations between the true mean values and the corresponding estimates under the fitted model and is computed for 50 evenly-spaced points along the domain of $x$.
} 
of orders 10, 2 (quadratic), 1 (linear), and 0 (null). In each setting, we generate data $(n=100)$ according to the following model:

$$
\begin{aligned}
& y_{i}=\alpha+\beta_{1} x_{i}+\beta_{2} x_{i}^{2}+\ldots+\beta_{10} x_{i}^{10}+\varepsilon_{i} \\
& \text { where } \varepsilon_{i} \stackrel{i i d}{\sim} N(0,1) \text { and } x_{i} \stackrel{i i d}{\sim} \operatorname{unif}(0,1)
\end{aligned}
$$

In order to compare many possible functional forms (for the high-order and 2-order settings), the $\beta_{j}$ parameters were randomly generated. For the high-order setting, we drew $\theta_{1}, \theta_{2}, \ldots, \theta_{10} \sim N(0,1)$, then scaled them so their magnitude sums to $10 ; \beta_{j}=10 * \theta_{j} / \sum\left|\theta_{i}\right|$. The same technique was used in the quadratic generating model, except only for $j \in\{1,2\}$; all other parameters were set to 0 . In the linear case, $\beta_{1}=10$, and in the null setting, $\beta_{j}=0 \forall j$. The sole covariate $x$ follows a standard uniform distribution within each simulation.

For model fitting, we utilize the cumulative SRL method with all terms up to the 10th order. We compare this model fit with the LOESS smoother (loess() in the stats package (R Core Team, 2020)) and with a smoothing spline (the gam() and $\mathbf{s}()$ functions from the mgcv package (Wood, 2011)). The default settings are used for these functions. The cumulative SRL is tuned using repeated $(r=5)$ 10-fold cross-validation with $\gamma \in\{0,0.5,1\}^{6}$. The simulations are repeated 1,500 times. Models are evaluated on the basis of the RMSE of estimation on $n=10,000$ new randomly sampled observations, presented in Figure 3. We find that the cumulative SRL achives similar performance to its LOESS and spline alternatives. Importantly, in contrast to alternatives, the cumulative SRL performs very well for the lower order and null models, predicting new values almost as well as the oracle model. This relative improvement is the result of overfitting of the alternative methods. SRL's superlative performance in the null setting matters to a great extent in the high-dimensional setting where we expect many null relationships. For the $10^{t h}$-order generating model, the poor performance of the "full" model, despite it being technically correctly specified, is a mark of the fact that there is high correlation among the polynomials of $x$, which inflates the variance of the estimated regression coefficients.

These simulation studies suggest that, at least for functional forms well-represented by polynomials, the cumulative SRL will do well to fit the relationship while also sifting through many null relationships. For other functional forms not well-represented by polynomials, or when the covariate has a high amount of skew, the cumulative SRL will not perform as well as spline/kernel alternatives. However, for the case of covariate skew, a normalizing transformation on the covariate prior to expanding the polynomial can mitigate this effect. We have developed software in a separate work that can adequately and robustly perform these normalizations (Peterson and Cavanaugh, 2020; Peterson, 2021).

\footnotetext{
${ }^{6}$ The $\gamma$ options of $0,0.5$, and 1 represent a minimal set of possible ranked sparsity settings for additional penalization for higher-order polynomials from none $(\gamma=0)$ to strong $(\gamma=1)$.
} 


\subsection{Interactions Simulation Study}

\subsubsection{Simulation Setup}

While we have shown how the SRL can compete with other smoothing techniques in the one-dimensional setting, the main benefits of the SRL methodology are present in the medium-to-high dimensional setting where model selection must take place. We set up a more extensive simulation in the context of interactions, comparing the SRL's performance to that of the glinternet method, the all-pairwise lasso (APL), and the lasso with only the main effects included (LS0).

Let $\mathbb{X}=\left[X, X^{\odot 2}\right]$ refer to the column combination of the main covariates (an $n \times p$ matrix), followed by their element-wise product values $\left(n \times\left(\begin{array}{l}p \\ 2\end{array}\right)\right)$. We wish to fit the linear model $\boldsymbol{y}=\mathbb{X} \boldsymbol{\beta}+\boldsymbol{\varepsilon}$, where we partition $\boldsymbol{\beta}^{T}=\left[\boldsymbol{\beta}_{1}^{T}, \boldsymbol{\beta}_{2}^{T}\right]$ to correspond with our notation for $\mathbb{X}$. In the usual case, where interactions are not considered, it is assumed that $\boldsymbol{\beta}_{1}$ is the only parameter vector with nonzero components. One would expect that this assumption helps in situations where the true generating model is, in fact, linear in the main covariates with no active interactions. However, what if there are nonzero components in the other parameter vector? We will investigate.

In the simulations to follow, we take $n=300$, and $p=20$, and we generate each element in $X$ as independent uniform $(0,1)$ random variables. The reason for independence in these predictors is to allow simple interpretations of the selection results (with correlated predictors, what constitutes a false discovery or a false negative is less well-defined). We investigate predictive performance in the setting of correlated features in the supplemental work, and mention the take-aways in this paper's discussion. We set the number of nonzero main effects $(s)$ in $\boldsymbol{\beta}_{1}$ as $s=5$. In order to generate our $\boldsymbol{\beta}$ coefficients in such a way that a large set of possible relationships are considered, we use scaled normal random variables as our "active" (i.e. nonzero) parameters in $\beta$. Specifically, we consider 11 generative settings of interest corresponding to the number of active interactions $b$, and the algorithm to generate the nonzero (active) coefficients is comprised of the following steps:

For $b \in\{0,1,2, \ldots, 10\}$ :

- Draw $\theta_{1}, \theta_{2}, \ldots, \theta_{s} \sim N(0,1)$

- Compute scaled main effects $\beta_{1 j}=10 * \theta_{j} / \sum\left|\theta_{i}\right|$

- Generate $b$ standard normal variables $\left\{\phi_{1}, \ldots, \phi_{b}\right\}$

- Select the index of active interactions $j$ according the rules outlined in the following paragraph

- Set $\beta_{2 j}=10 * \sqrt{\frac{12}{7}} \phi_{j} / \sum\left|\phi_{i}\right|^{7}$

\footnotetext{
${ }^{7}$ Scaling by $\sqrt{\frac{12}{7}}$ accounts for the difference in variability between uniform random features and their interactions.
} 
The generating models were not necessarily strongly hierarchical. In particular, each simulation was configured according to Chipman's paradigm; strong hierarchy was most probable, weak hierarchy less so, and non-hierarchy least so. Given $s=5$ and $p=20$, there are $\left(\begin{array}{l}s \\ 2\end{array}\right)=10$ candidate interactions that would yield a strongly hierarchical model, $s(p-s)=75$ that would yield a weakly hierarchical model, and $\left(\begin{array}{c}p-s \\ 2\end{array}\right)=105$ that would yield an non-hierarchical model. Within a simulation, each active interaction effect (if there were any) was drawn at random from these bins of strong, weak, or non-hierarchical candidate effects with probabilities $0.7,0.2,0.1$, respectively.

In order to fit these models, we consider four modeling frameworks: LS0 (lasso on original terms only), APL (lasso with original and all pairwise interaction terms), SRL (sparsity-ranked lasso with original and all pairwise interaction terms), and GLN (glinternet model). For each framework, the optimal $\lambda$ is selected with 10-fold CV, and then that tuned model is used to predict 10,000 new randomly sampled observations. The $\gamma$ parameter for SRL is fixed to 0.5 , corresponding to an equal contribution of prior information from the main effects and the interaction effects. This process (including the new generation of $\beta_{i j}$ terms) is repeated 1,000 times in order to check the models' predictive and selective performance. For the former, we use the RMSE of prediction on newly generated data to compare the predictive accuracy of the final models ${ }^{8}$. For the latter, we use the false discovery rate (FDR), the mean number of Type I errors, and the mean number of Type II errors, examining these quantities both collectively and separately for interactions and main effects. In particular, these selection metrics for interaction terms loosely measure the "transparency" and "interpretability" of the various models; models with many false discoveries in the interaction effects are needlessly opaque.

\subsubsection{Simulation Results}

The predictive performance of the models across all simulations is shown in Figure 4. Although the LS0 model demonstrates a very slight gain in predictive performance if the true model has no interactions, it also exhibits a marked loss in performance when any active interactions are present. The APL model performs comparably better than the LS0 model when any interactions are present, but performs much worse in the no-interaction case. SRL performs much better than either the APL or GLN in the no-interaction case. SRL and GLN have similar predictive performance to each other when active interactions are present, both performing much better than either the APL or LS0. In the supplement, we show how other correlation structures exhibit similar results; in fact, SRL's relative performance compared to GLN and APL is sometimes even better with higher correlation among features. In situations where the SRL performed worse than other methods (such as the compound symmetry correlation matrix with feature correlation $\rho=0.5$ ), selecting the

\footnotetext{
${ }^{8}$ The RMSE of prediction is based on the sum of the squared deviations between each new observation and the corresponding predicted value under the fitted model.
} 
optimal $\gamma \in\{0,0.5, \infty\}$ using CV still performed comparably to the best alternative.

The plots in Figure 5 show model selection information for each framework. When the true model has no active interactions, the LS0 and the SRL methods look very similar in terms of FDR and the mean number of Type I/II errors. In this same setting, the GLN and APL models have a much higher FDR and mean number of Type I errors; this is driven by the tendency of these models to select too many false interactions (rendering selected models unnecessarily opaque). For all of the generative settings, the overall FDR for the APL is very high, and it is driven disproportionately by a high FDR in the interaction effects. The GLN method also exhibits this differential expression of the FDR among main and interaction effects, though to a lesser degree. This difference is further seen in the number of Type I errors; the higher FDR in the interaction effects translates to many more Type I errors for GLN and APL than for SRL. The SRL method maintains approximately the same number of Type I errors in the interaction effects and the main effects for $b \geq 4$. This improvement in FDR/Type I error rate exhibited by SRL compared to GLN is balanced out by a slightly higher mean number of Type II errors, a disparity which grows with the number of active interactions. In summary, when interactions are especially sparse, the SRL outperforms every other method in terms of prediction and selection. Otherwise, the SRL and GLN perform comparably to one another in terms of prediction, although the SRL produces more interpretable/transparent models by admitting fewer unnecessary (false) interactions.

\section{Application: Gene-Environment Interactions}

\subsection{Background}

We wish to show how SRL methods can be used in the context of genetic data, specifically for the purpose of detecting important gene-environment interactions. Gene-environment interactions make sense biologically, but unfortunately, they are very difficult to detect in practice. With high-dimensional data, the detection of any meaningful association is sufficiently challenging, yet looking for interactions with high-dimensional data is akin to searching for several needles in tens of thousands of haystacks. We utilize a study that collected data on 442 patients with lung cancer (adenocarcinoma) (Shedden et al., 2008). For each patient, the investigators observed the time of death or censor (the primary outcome), 22,283 gene expression measurements taken from a sample of the lung cancer tumor, and some clinical covariates: sex, race, age, whether the patient received chemotherapy, smoking history, and cancer stage. The main outcome of overall survival is presented in Figure 6. 


\subsection{Methods}

In order to fit models with gene-environment interactions, we take our outcome $(\boldsymbol{y}, \boldsymbol{d})$ as the time until death/censor and the death indicator, respectively. We model the hazard of death using a Cox proportional hazards model with our predictors being comprised of the genetic and clinical covariates described in the previous section denoted by $X$ and $Z$, respectively. For our purposes, we are only interested in interactions that may occur between $X$ and $Z$ or within $Z$; we do not look for interactions that occur within $X^{9}$. Since the outcome is time-to-event, we substitute the partial likelihood for the Cox regression model into the objective function, taking the place of the least squares term.

We investigate the performance of three candidate modeling frameworks: the lasso using only main effects (LS), the sparsity-ranked lasso (SRL), and the all-pairwise lasso (APL). Within the SRL framework, we set $\gamma=0.5$, and investigate three different penalty schema. Since our features consist of both clinical and genetic covariates, we treat these as two separate covariate groups of different sizes in a model we abbreviate as SR0, which does not include any interactions (but still uses the SRL framework). SR1 refers to the SRL approach for both the main and interaction effects with proportional weighting. Finally, SR2 refers to the cumulative SRL, wherein the penalty increases cumulatively for clinical covariates, genetic covariates, and their interactions (in that order).

The first step in the modeling process is to split the data $\mathbb{X}=[\boldsymbol{y}, \boldsymbol{d}, X, Z]$ randomly into a training set $\mathbb{X}_{\text {train }}(n=342)$ and a test set $\mathbb{X}_{\text {test }}(n=100)$. Second, based on $\mathbb{X}_{\text {train }}$, we use repeated $(r=10)$ cross-validation $(k=10)$ to tune each of the aforementioned models (with respect to $\lambda$ ). At this stage, we also select the optimal modeling structure. Third, we use the optimally tuned model within each modeling structure to predict outcomes on the test set $\mathbb{X}_{\text {test }}$, comparing performance between the models and confirming that the optimal structure we selected in the prior step performed the best on $\mathbb{X}_{\text {test }}$. Finally, we re-fit and re-tune the optimal modeling structure using the full data $\mathbb{X}$ in order to interpret the best final model.

In order to assess predictive efficacy for cross validation, we use the expected extra-sample Cox partial deviance, estimated as described in the ncvreg documentation (Breheny and Huang, 2011). While this measure is difficult to interpret in an absolute sense, it can be effective in assessing predictive accuracy in a relative sense. We also calculate an estimate of the out-of-sample $R^{2}$ based on the deviance, and we measure both accuracy measures using the test set as well as the CV process.

As a final purely visual assessment of predictive performance, we categorize individuals from the test data into three categories based on their expected risk score (low-risk, medium-risk, or high-risk). The cut points

\footnotetext{
${ }^{9}$ Strictly speaking, the clinical covariates are not all environmental, but we decided to include all of their interactions as candidates out of interest. However, selected interactions should be considered in their proper context and accordingly labelled using appropriate terminology.
} 
Table 1: Estimated predictive performance (mean deviance and Cox-Snell $R^{2}$ ) calculated using extra- and out-of-sample data broken down by modeling framework. Cross-validated values are estimated with 10 -folds and 10 repeats.

\begin{tabular}{lrrrrrr}
\hline & \multicolumn{2}{c}{ Cross-Validation } & & \multicolumn{2}{c}{ Test set } \\
\cline { 2 - 3 } \cline { 6 - 7 } & Deviance & $R^{2}$ & & Deviance & $R^{2}$ \\
\hline Lasso with only main effects (LS) & 10.35 & 0.060 & & 7.77 & 0.141 \\
SRL with only main effects, proportional weights (SR0) & 10.20 & 0.188 & & 7.70 & 0.196 \\
SRL with interactions, proportional weights (SR1) & 10.18 & 0.205 & & 7.72 & 0.184 \\
SRL with interactions, cumulative weights (SR2) & 10.22 & 0.177 & & 7.69 & 0.204 \\
All-pairwise lasso (APL) & 10.40 & 0.013 & & 7.86 & 0.054 \\
\hline
\end{tabular}

are set to be the 33rd and 67 th percentile of the linear predictions on the test set, which could vary across methods. Then, using the test set, we plot Kaplan-Meier (KM) curves for each method stratified by the test set's predicted risk score categories. More separation among those stratifications on the KM plot means better predictive performance; such delineation indicates that the model is doing a good job of classifying high-, medium-, and low-risk patients in the test set.

\subsection{Results}

The estimated extra- and out-of-sample Cox partial deviance and Cox-Snell $R^{2}$ by model is shown in Figure 7 and Table 1. We find APL performed quite poorly, which indicates that the consideration of pairwise interactions, without accounting for ranked sparsity, is not a good idea. The LS method performed only slightly better, which indicates that penalizing the genetic and clinical covariates equally may not be advised either. The relatively strong performance of SR0, SR1, and SR2 indicates that the sparsity-ranked lasso achieves a satisfactory middle ground. Since these SRL models all perform similarly, and none of them select any interactions (see Table 2), we have little to no evidence that any prominent gene-environment interactions are capable of being discovered.

In Figure 8, we show the categorization efficacy of each model using the test data set. SR2 is omitted here because its performance is very similar to SR0. In the plots, we note that the LS and the APL models did a good job classifying high-risk patients, but did not distinguish well between medium- and low-risk patients. The SR0 and SR1 models seem to have done a relatively good job classifying individuals in the test set, which is most likely due to the handling of the clinical covariates (SRL is shrinking the clinical variables relatively less than the LS model).

After re-fitting and re-tuning each model to the entire data set, we examine the number of selections (S) and the sum of the magnitude of the standardized coefficients by covariate group for each optimally tuned model in Table 2. Evidently, the LS model found most of its signal from the genetic covariates; 43 of 
Table 2: The number of selections (S) and the sum of the magnitude of the standardized coefficients by covariate group for each (optimally tuned) model. Tuning of $\lambda$ was accomplished with 10 -fold cross-validation with 10 repeats, and $\gamma$ was set to 0.5 . LS refers to the lasso on the original covariates, SR0 refers to the SRL with proportional penalties on clinical and genetic covariates, SR1 refers to the SRL with interactions and proportional penalty weights, SR2 refers to the SRL with interactions and cumulative penalty weights, and APL refers to the all-pairwise lasso.

\begin{tabular}{|c|c|c|c|c|c|c|c|c|c|c|}
\hline & \multicolumn{2}{|c|}{ LS } & \multicolumn{2}{|c|}{ SR0 } & \multicolumn{2}{|c|}{ SR1 } & \multicolumn{2}{|c|}{ SR2 } & \multicolumn{2}{|c|}{ APL } \\
\hline & $\mathrm{S}$ & $\|\beta\|_{1}$ & S & $\|\beta\|_{1}$ & S & $\|\beta\|_{1}$ & S & $\|\beta\|_{1}$ & $\mathrm{~S}$ & $\|\beta\|_{1}$ \\
\hline Clinical & 3 & 0.134 & 6 & 0.938 & 6 & 0.926 & 6 & 0.935 & 1 & 0.013 \\
\hline Genetic & 43 & 1.133 & 42 & 0.857 & 31 & 0.583 & 42 & 0.786 & 6 & 0.229 \\
\hline Env-Env & 0 & 0.000 & 0 & 0.000 & 0 & 0.000 & 0 & 0.000 & 0 & 0.000 \\
\hline Gene-Env & 0 & 0.000 & 0 & 0.000 & 0 & 0.000 & 0 & 0.000 & 5 & 0.041 \\
\hline
\end{tabular}

Table 3: Number of selected coefficients common among each method.

\begin{tabular}{lccccc}
\hline & LS & SR0 & SR1 & SR2 & APL \\
\hline LS & 46 & 34 & 27 & 34 & 7 \\
SR0 & & 48 & 36 & 48 & 7 \\
SR1 & & & 37 & 36 & 7 \\
SR2 & & & & 48 & 7 \\
APL & & & & & 12 \\
\hline
\end{tabular}

which had nonzero coefficients. Only 3 clinical covariates were selected, and the combined magnitude of the standardized coefficients $\left(\|\beta\|_{1}\right)$ was only 0.134 . SR0, on the other hand, found the majority of the signal to lie in the six clinical covariates $\left(\|\beta\|_{1}=0.938\right)$, though it still found a good amount of signal (0.857) in 42 of the genetic covariates. Neither SR1 nor SR2 selected any gene-environment interactions. SR1 found less signal in the genetic variables than SR0 - this indicates that the addition of interaction terms necessitated a higher amount of shrinkage in the main effects (particularly the genetic main effects). For SR2 however, since the coefficients are being penalized in a cumulative fashion, the amount of signal is very similar to SR0 when no interactions were considered. APL, although having discovered 5 gene-environment interactions, is clearly not able to find much signal at all; it is shrinking all of the effects considerably. These results taken together indicate that there are no informative gene-environment interactions, and that the clinical variables should be penalized proportionally less than the genetic variables.

In Table 3, we show how many selected variables were shared for each model selection method. There was very high agreement in the SR models, and in fact perfect agreement between SR0 and SR2 (they selected all of the same variables). SR1 selected only one covariate that was not selected by SR0 or SR2, a feature called "checkpoint kinase 1," although its estimated coefficient was very small.

Finally, we will interpret the SR0 model, which was very similar to the SR2 model. In terms of the 
estimated hazard ratios (HRs), the most protective effect we found was for those in the "never smoked" group $(\mathrm{HR}=0.74)$. We found two clinically significant protective gene expressions: FAM117A $(\mathrm{HR}=0.89)$, and CTAGE5 (HR = 0.90). We found harmful effects if subjects were white $(\mathrm{HR}=1.33)$, male $(\mathrm{HR}=1.32)$, or had chemotherapy $(\mathrm{HR}=1.94)$. Additionally for every 10 year increase in age, the hazard increases by a multiplicative factor of 1.44. Interestingly, the clinical coefficients in this model are similar to the estimates from the model with only clinical covariates. Oddly, the identified important gene expressions selected by SR0 were not used as classifiers in the original paper. Note that since this was not a randomized controlled trial, these effects are not indicative of causal relationships; in particular, the high HR on chemotherapy status does not indicate that chemotherapy was harmful.

\section{Discussion}

\subsection{Strengths and Weaknesses of the SRL}

We have shown that the sparsity-ranked lasso performs relatively well for selecting transparent models. Whereas other methods for selecting polynomials and/or interactions tend to select overly opaque models (models with high-order relationships that are difficult to interpret), SRL naturally selects models that have more main effects and fewer "complicating" terms. In other words, the SRL limits the tendency to select too many interactions, and controls the number of false discoveries among interactions to be close to the same or less than that in the main effects. Therefore, the SRL is a technique that can be utilized and trusted to select from interactions and polynomials without yielding overly convoluted interpretations.

Since many authors have already contributed to the problem of selecting from all possible interactions, discussion of the SRL compared to these competing methods is warranted. One major benefit to the SRL is that it can be applied to survival outcomes; at the time of writing, all of the competing methods we have mentioned are supported by open-source software packages, but none can handle survival outcomes to our knowledge. Thanks to the versatility of the ncvreg package, the SRL method can be used for binomial, continuous, survival, or Poisson outcomes (Breheny and Huang, 2011). Further, in sparseR, sparsity-ranked versions of non-convex regularization methods such as the Minimax Concave Penalty (MCP) (Zhang, 2010) and the Smoothly Clipped Absolute Deviations (SCAD) penalty (Fan and Li, 2001) are also feasible and implementable. We have shown that these non-convex methods work quite well (empirically) in this paper's supplement (Figures S7, S8). Another benefit of the SRL to consider is the computational speed; glinternet has been shown to be 10-10000 times faster than hierNet, and yet our method is quite a bit faster than glinternet (at least for our simulation settings, see supplemental Figures S9, S10). This speed-up does not 
seem to change as the sample size increases, and it is especially noticeable when cross-validation is employed to tune the models.

Perhaps most importantly, we have found that SRL works better than glinternet (in terms of prediction accuracy and the false discovery rate) when there are no interactions or when interactions are especially sparse. This strength suggests another important benefit to the SRL producedure; it can be worthwhile, convenient, and straightforward to extend the SRL to examine higher order interactions (and polynomials). As opposed to competing methods, the SRL will not heavily inflate the number of Type I errors in the course of such an investigation, even as number of $k$-order interactions increases combinatorically with $k$.

One large weakness to the SRL is that it requires storage of a potentially large matrix of interactions. However, recent advances in the scalability of regularization algorithms such as the biglasso package (Zeng and Breheny, 2021) are applicable to the SRL as well. Another weakness that the SRL shares with other regularization procedures is that the optimal mechanism of formal inference is unclear. It is possible to extend recent advances in the marginal false discovery rate (mFDR) (Breheny, 2018; Miller and Breheny, 2019) to the SRL framework, and this method is currently included in the sparseR package. Yet whether or not this method is optimal for formal inference and whether the mFDR works well for ranked-sparsity settings remains an area of future research. Finally, one often unaddressed issue with using regularization to search for important interactions is that the model fit is sensitive to the choice of origin among the covariates; in particular, the method is not invariant to changes of location, such as centering, in the covariates. The tutorial for sparseR goes more into detail about what can be done in circumstances where the best origin location is unknown ahead of time. One solution is to use our ranked-sparsity-based information criterion RBIC (Peterson, 2019), which is invariant to location changes in the covariates, to search for and select an optimal model, comparing this fit with the estimates from the regularization procedure.

In the course of our exploration, some of our results indicate that the SRL will not perform as well as competitors in certain situations. This relatively poor performance was seen when using the cumulative SRL for polynomials in settings with highly skewed covariates or when the functional forms cannot be well-represented by polynomials, in which circumstances other smoothers tend to work better. Also, the performance of the SRL for interactions relative to glinternet seems to depend on the hierarchical configuration of the generating model; glinternet can perform slightly better than the SRL when the true model is strongly hierarchical, whereas the SRL method performs better when the true model is weakly or non-hierarchical. Their relative predictive performance depends to an extent on the mix of strong, weak, and non-hierarchical active interactions, and more research is needed to determine exactly how and why this is the case.

Finally, while we have motivated intuitive guidelines for the selection of $\gamma$, future work should investigate the practicality and utility of optimizing the choice of $\gamma$ with respect to predictive accuracy. We incorporated 
$\gamma$ in the formulation of the SRL for two reasons: (1) to show that the original lasso can be written as a special case of the SRL when $\gamma=0$, and (2) to explore the apparent benefits to the cumulative SRL for polynomials from additional tuning of its weighting scheme. For this reason, we performed a minor amount of tuning for $\gamma$ when applying the cumulative SRL in this work (showing that BIC or cross-validation can be used). Outside of the cumulative SRL, e.g. in our real data analysis, we opted for fixing $\gamma=0.5$ for simplicity and because we intended each covariate group to contribute the same amount of prior information for SR0 and SR1. For SR2, we acknowledge that further tuning of $\gamma$ may yield slightly better results, but would then be less comparable to SR0 and SR1.

\subsection{Other Applications of the SRL}

Though not the primary focus of this paper, the SRL has wide reaching applications outside of interaction and polynomial feature selection. We have developed SRL methods for automated autoregressive (AR) order selection for time series data (Miller et al., 2019), finding the procedure particularly helpful for seasonal time series with uncertainty in the seasonal period. Additionally, we have utilized the SRL in conjunction with adaptive out-of-sample time series regression methods to incorporate past states of a model into the fitting of current or future models via varying penalization weights. Finally, we have extended the SRL into what we call "ranked cost" contexts, wherein candidate covariates have quantifiably different costs of data collection. In this setting, if any correlation exists among these features, the SRL can simultaneously optimize for predictive accuracy and the costs of future data collection; it produces the least costly model that can still predict as well as the optimal model. Our exploration of these extensions is still ongoing, but initial results have been promising.

In the context of gene-environment interactions, single nucleotide polymorphism (SNP) data are frequently used rather than gene expression data. SNP data are ordinal/categorical, and tend to have very high amounts of correlation. While further exploration of the performance of the SRL in the context of ordinal/categorical covariate data is warranted, we postulate that ranked sparsity methods would be a fruitful approach. In particular, ranked sparsity can be paired with other existing regularization approaches that work well for highly correlated data such as the elastic net, which is implemented in the sparseR package. In these highly-correlated settings, additional (minor) tuning of the SRL between $\gamma \in\{0,0.5, \infty\}$ can improve its relative performance to glinternet and the APL (Figures S2-S6) ${ }^{10}$. Further, for SNP data, a common simplifying assumption is that each SNP count relates linearly to the outcome (treating these covariates as numeric rather than categorical). The cumulative SRL approach for polynomials can navigate a powerful

\footnotetext{
${ }^{10}$ This minor tuning simply involves picking the best performing model between the APL $(\gamma=0)$, SRL $(\gamma=0.5)$, and LS0 $(\gamma=\infty)$; no additional $\mathrm{CV}$ is necessary.
} 
middle-ground between these qualitative/quantitative extremes. While it treats the SNP count as numeric and prefers linearity, when strong evidence of nonlinearity is observable, the cumulative SRL approach will guardedly introduce polynomial terms to the active feature set. Conversely, in the absence of such evidence, the approach will not yield an overabundance of false discoveries.

\subsection{Conclusion}

The ranked sparsity framework implements a broader definition of Occam's Razor where a model's simplicity is not purely equated to parsimony; it is also tied to the model's transparency and interpretability. The sparsity-ranked lasso provides an effective and fast approach for selecting from derived variables such as interactions or polynomials. As opposed to other methods of interaction selection, the SRL does not select an unreasonable number of false interaction effects and it does not overly shrink the main effects.

\section{References}

Akaike, H. (1974) A new look at the statistical model identification. IEEE Transactions on Automatic Control, 19, 716-723.

Bien, J., Taylor, J. and Tibshirani, R. (2013) A lasso for hierarchical interactions. The Annals of Statistics, 41, 1111-1141.

Bogdan, M., Frommlet, F., Biecek, P., Cheng, R., Ghosh, J.K. and Doerge, R. (2008) Extending the modified Bayesian information criterion ( $\mathrm{mBIC}$ ) to dense markers and multiple interval mapping. Biometrics, 64, 1162-1169.

Boulesteix, A.-L., De Bin, R., Jiang, X. and Fuchs, M. (2017) IPF-lasso: Integrative-penalized regression with penalty factors for prediction based on multi-omics data. Computational and Mathematical Methods in Medicine, 2017, 7691937.

Breheny, P.J. (2018) Marginal false discovery rates for penalized regression models. Biostatistics, 20, 299-314.

Breheny, P. and Huang, J. (2011) Coordinate descent algorithms for nonconvex penalized regression, with applications to biological feature selection. Annals of Applied Statistics, 5, 232-253.

Chen, J. and Chen, Z. (2008) Extended Bayesian information criteria for model selection with large model spaces. Biometrika, 95, 759-771.

Chipman, H. (1996) Bayesian variable selection with related predictors. The Canadian Journal of Statistics / La Revue Canadienne de Statistique, 24, 17-36.

Choi, N.H., Li, W. and Zhu, J. (2010) Variable selection with the strong heredity constraint and its oracle property. Journal of the American Statistical Association, 105, 354-364.

Fan, J. and Li, R. (2001) Variable selection via nonconcave penalized likelihood and its oracle properties. Journal of the American Statistical Association, 96, 1348-1360.

Friedman, J., Hastie, T. and Tibshirani, R. (2010) A note on the group lasso and a sparse group lasso. preprint: arXiv:1001.0736.

Hao, N., Feng, Y. and Zhang, H.H. (2018) Model selection for high-dimensional quadratic regression via regularization. Journal of the American Statistical Association, 113, 615-625.

Hurvich, C.M. and Tsai, C.-L. (1989) Regression and time series model selection in small samples. Biometrika, 76, 297-307.

Klau, S., Jurinovic, V., Hornung, R., Herold, T. and Boulesteix, A.-L. (2018) Priority-lasso: A simple hierarchical approach to the prediction of clinical outcome using multi-omics data. BMC bioinformatics, 19, 322 . 
Kuhn, M. and Wickham, H. (2019) recipes: Preprocessing tools to create design matrices. R package, available at https://CRAN.R-project.org/package=recipes.

Lim, M. and Hastie, T. (2015) Learning interactions via hierarchical group-lasso regularization. Journal of Computational and Graphical Statistics, 24, 627-654.

Mallows, C.L. (1973) Some comments on Cp. Technometrics, 15, 661-675.

Miller, R.E. and Breheny, P. (2019) Marginal false discovery rate control for likelihood-based penalized regression models. Biometrical Journal, 61, 889-901.

Miller, A.C., Peterson, R.A., Singh, I., Pilewski, S. and Polgreen, P.M. (2019) Improving State-Level Influenza Surveillance by Incorporating Real-Time Smartphone-Connected Thermometer Readings Across Different Geographic Domains. Open Forum Infectious Diseases, 6.

Peterson, R.A. (2019) Ranked Sparsity: A Regularization Framework for Selecting Features in the Presence of Prior Informational Asymmetry. PhD thesis, Department of Biostatistics, University of Iowa.

Peterson, R.A. (2021) Finding Optimal Normalizing Transformations via bestNormalize. The $R$ Journal, 13, 310-329.

Peterson, R.A. and Cavanaugh, J.E. (2020) Ordered quantile normalization: A semiparametric transformation built for the cross-validation era. Journal of Applied Statistics, 47, 2312-2327.

R Core Team. (2020) R: A Language and Environment for Statistical Computing. R Foundation for Statistical Computing, Vienna, Austria.

Schwarz, G. (1978) Estimating the dimension of a model. The Annals of Statistics, 6, 461-464.

Shedden, K., Taylor, J., Enkemann, S., Tsao, M., Yeatman, T., Gerald, W., et al. (2008) Gene expressionbased survival prediction in lung adenocarcinoma: A multi-site, blinded validation study. Nature Medicine, 14, 822-827.

Ternès, N., Rotolo, F., Heinze, G. and Michiels, S. (2017) Identification of biomarker-by-treatment interactions in randomized clinical trials with survival outcomes and high-dimensional spaces. Biometrical Journal, 59, 685-701.

Tibshirani, R. (1996) Regression shrinkage and selection via the lasso. Journal of the Royal Statistical Society: Series B, 58, 267-288.

Wang, M. and Wang, X. (2014) Adaptive lasso estimators for ultrahigh dimensional generalized linear models. Statistics \&3 Probability Letters, 89, 41-50.

Wood, S.N. (2011) Fast stable restricted maximum likelihood and marginal likelihood estimation of semiparametric generalized linear models. Journal of the Royal Statistical Society (B), 73, 3-36.

Zeng, Y. and Breheny, P. (2021) The biglasso Package: A Memory- and Computation-Efficient Solver for Lasso Model Fitting with Big Data in R. The R Journal, 12, 6-19.

Zhang, C.-H. (2010) Nearly unbiased variable selection under minimax concave penalty. The Annals of Statistics, 38, 894-942.

Zou, H. (2006) The adaptive lasso and its oracle properties. Journal of the American Statistical Association, 101, 1418-1429. 

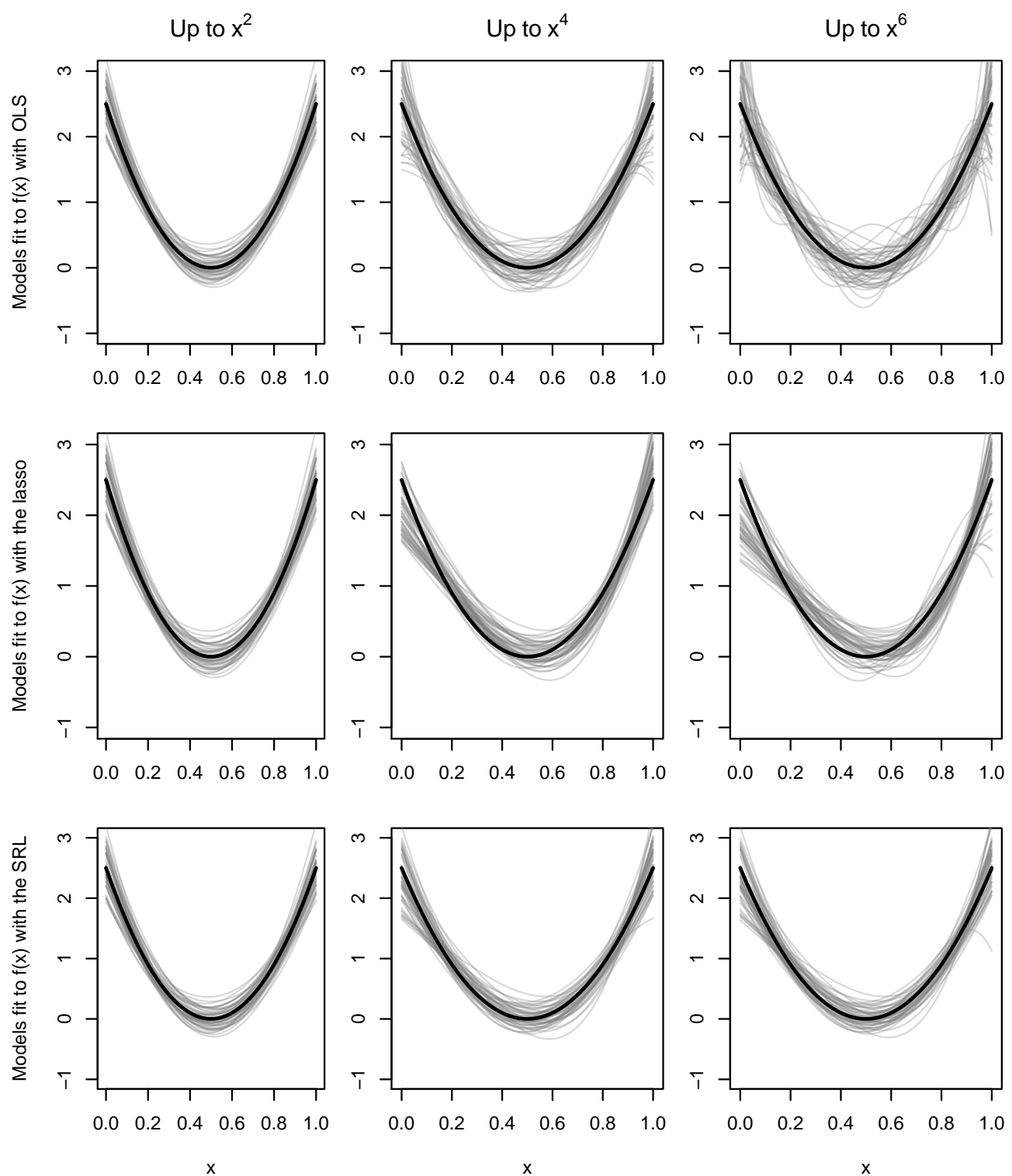

Figure 1: A simple simulation where 50 samples of size 100 are generated for $x$ and a response variable $y$ with the relationship $y=f(x)+N\left(0, .9^{2}\right)$. The black line represents the true $f$, and the grey lines represent 50 fits to the different samples. Models in the top three plots are fit using ordinary least squares (OLS); in the middle three plots, models are fit with the lasso; and on the bottom three plots, models are fit using the sparsity-ranked lasso (SRL). SRL and lasso models are tuned using BIC. The covariates included are the polynomials of $x$ up to $x^{2}$ (left) up to $x^{4}$ (center), and up to $x^{6}$ (right). 
Factor Increase in RMSE Relative to OLS (Up to $x^{2}$ )

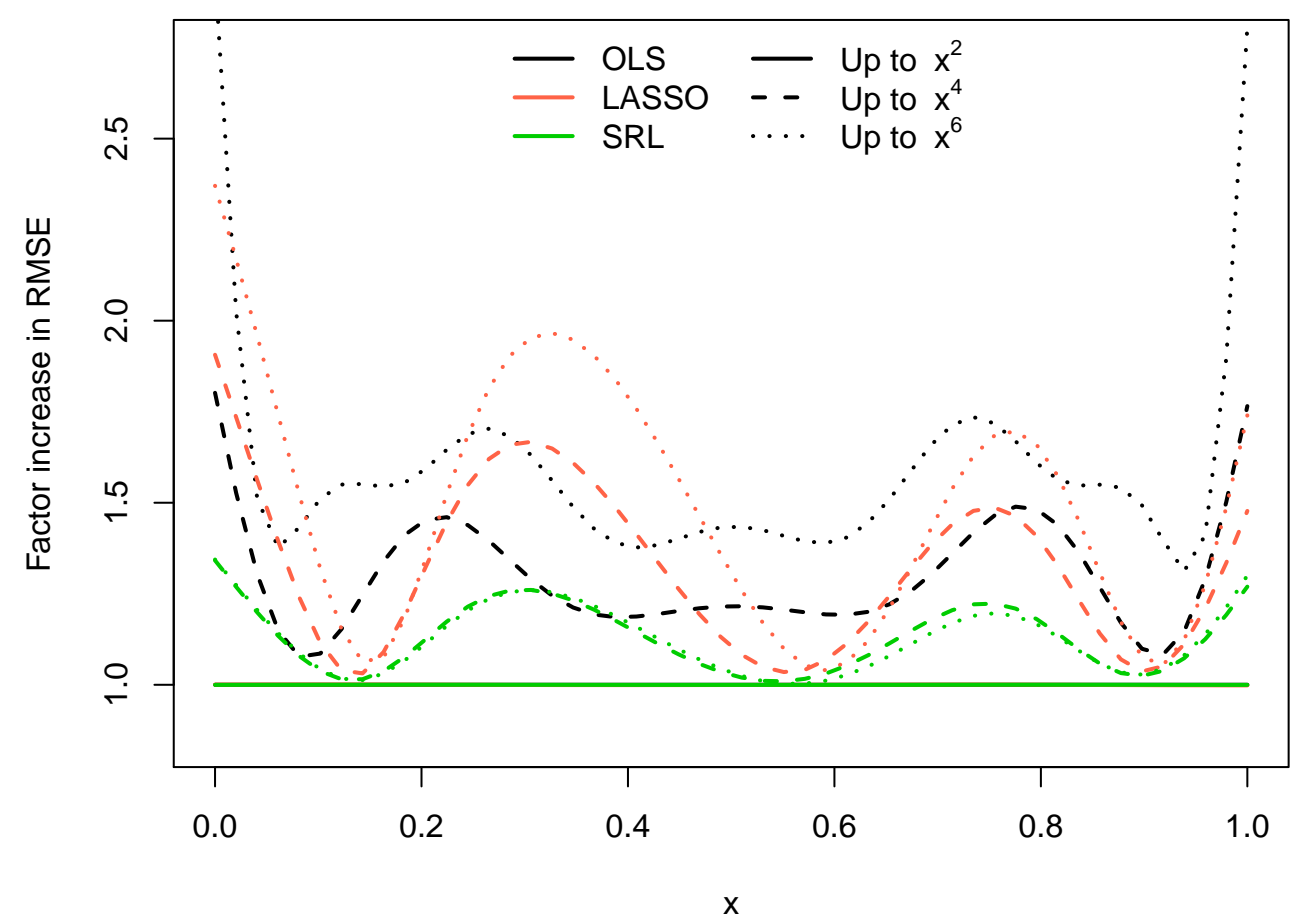

Figure 2: The expected increase in the root-mean-squared error (RMSE) for the ordinary least squares (OLS), lasso, and sparsity-ranked lasso (SRL) models relative to a baseline "oracle" OLS model that only includes the "true" variables $\left(x\right.$ and $\left.x^{2}\right)$. 
10-degree Model

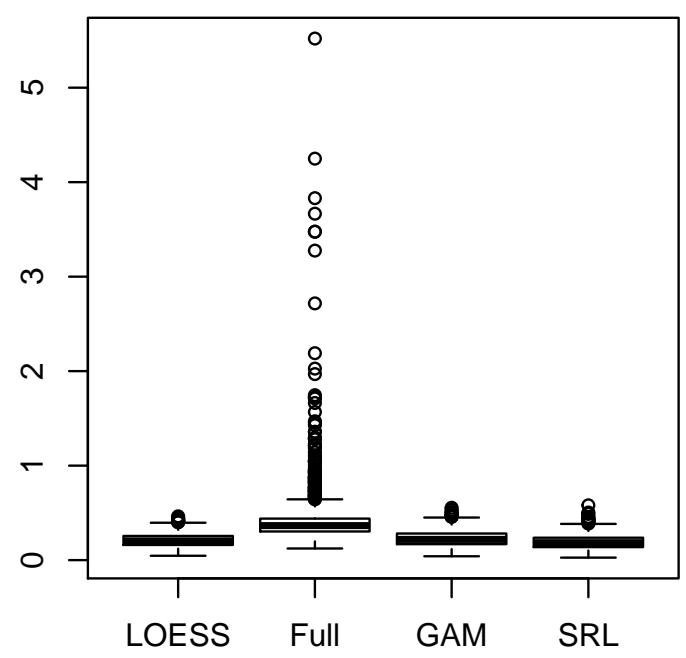

Linear Model

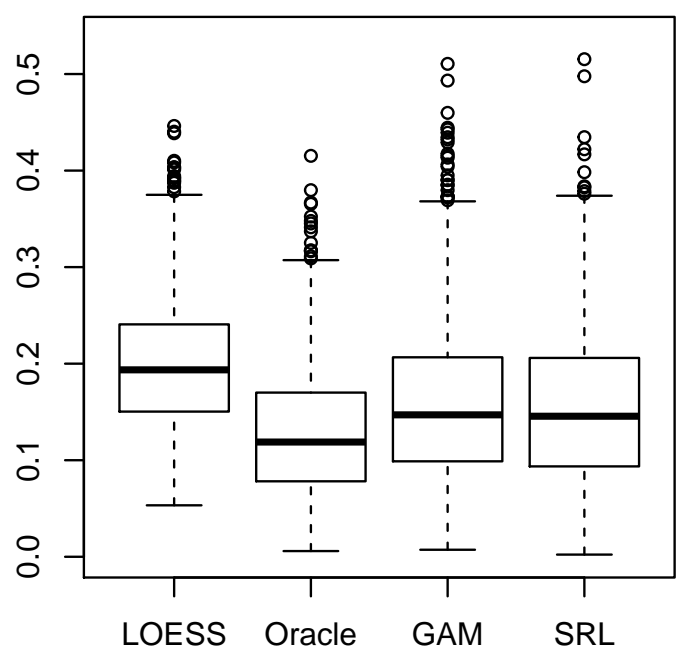

Quadratic Model

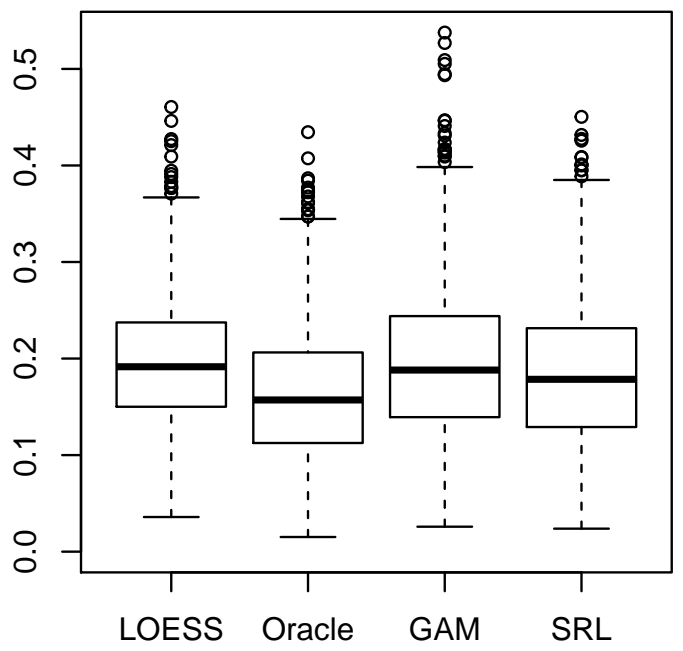

Null Model

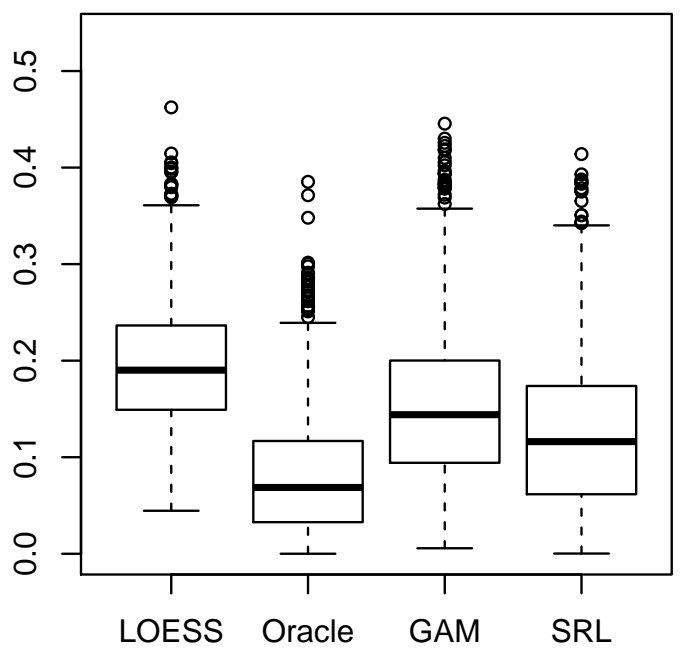

Figure 3: Performance of smoothing methods in describing a truly polynomial (or null) relationship between a single covariate and response. The RMSE within each simulation is plotted along the y-axis. SRL and lasso models are tuned using BIC. 
Factor Increase in RMSE Relative to SRL

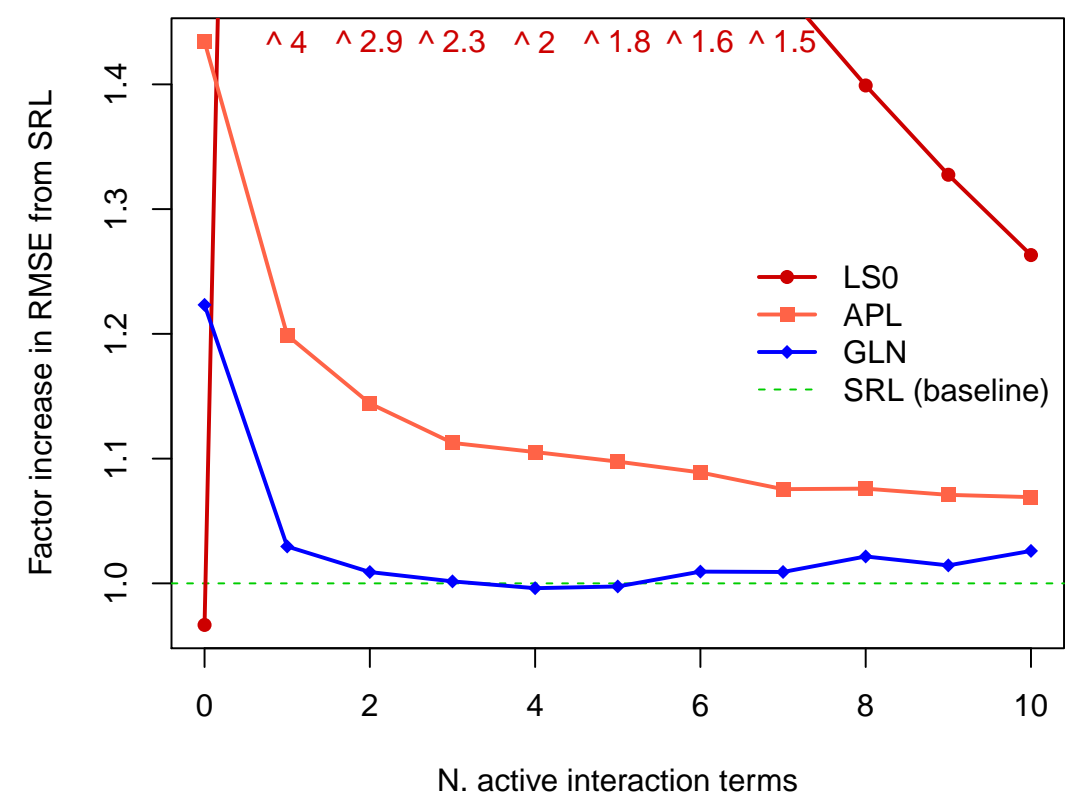

Figure 4: Predictive performance of various interaction fitting methods relative to SRL. LS0 refers to the lasso fit using only the original terms, APL refers to the lasso fit using the original terms and all pairwise interactions, SRL refers to the sparsity-ranked lasso fit with $\gamma=0.5$, and GLN refers to the glinternet model. For all models, $\lambda$ was tuned with 10-fold cross-validation. The "ح" notation refers to the values of the LS0 model that were too large to be clearly plotted next to the other curves. 


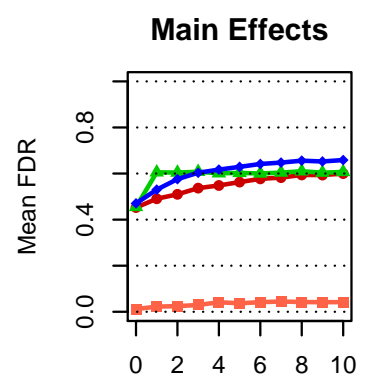

Interaction Effects
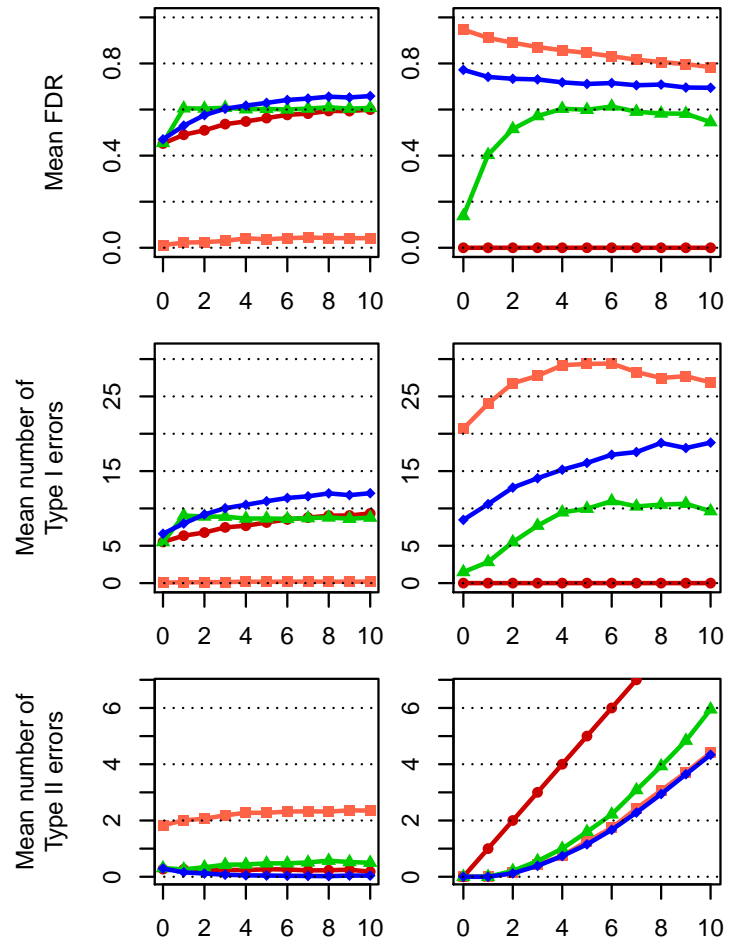

N. active interactions

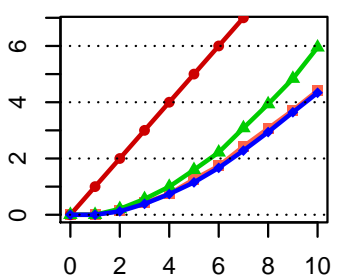

N. active interactions
All Effects
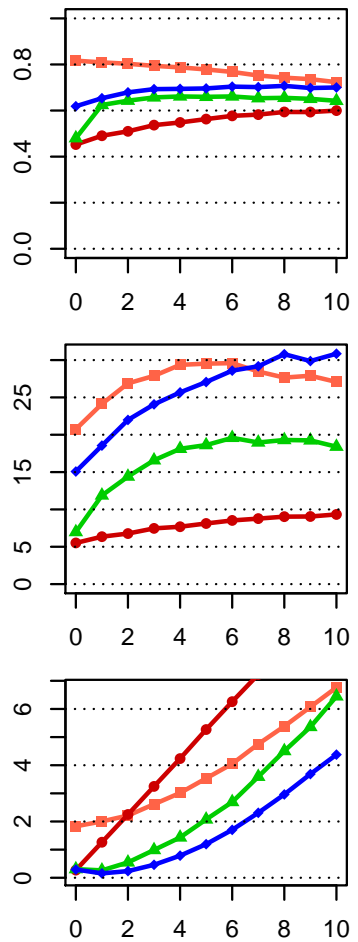

N. active interactions

Figure 5: Model selection performance of various interaction fitting methods. The top three plots show the mean FDR across simulations, the middle three plots show the mean number of Type I errors, and the bottom three plots show the mean number of Type II errors. The metrics are stratified into main-effects (left), interaction effects (center), and their combined/overall values (right). LS0 refers to the lasso fit using only the original terms, APL refers to the lasso fit using the original terms and all pairwise interactions, SRL refers to the sparsity-ranked lasso fit with $\gamma=0.5$, and GLN refers to the glinternet model. For all models, $\lambda$ was tuned with 10 -fold cross-validation. 


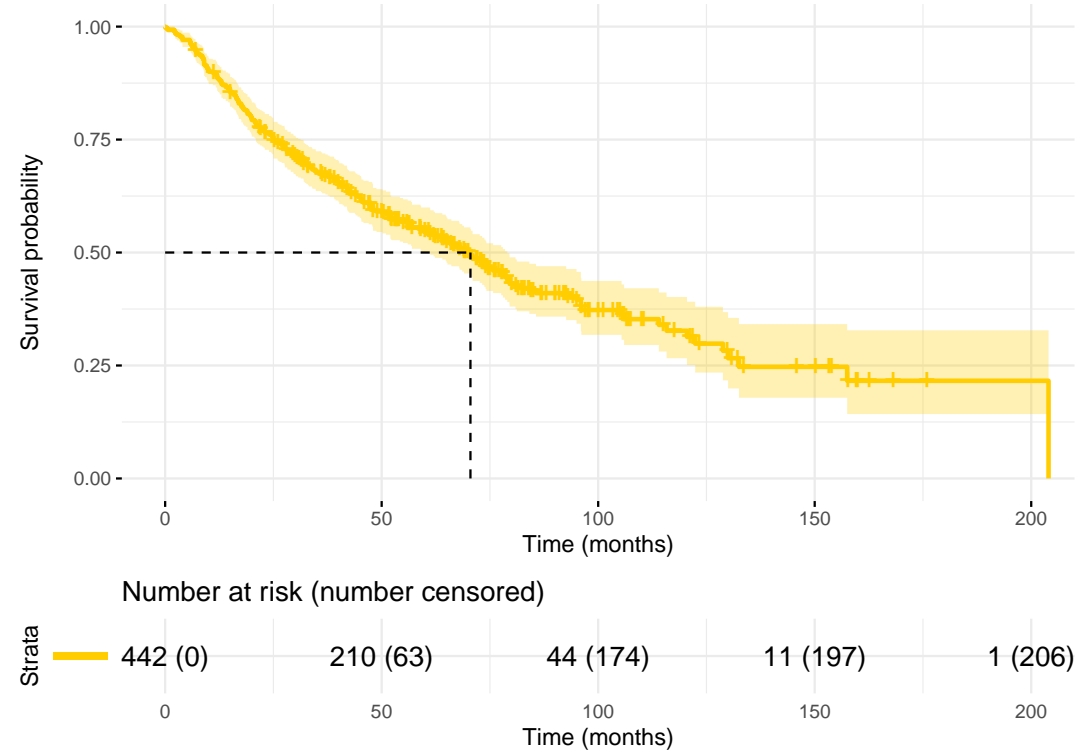

Figure 6: Kaplan-Meier curve for Shedden data; survival time is the primary outcome of the study for which we build regularized Cox regression models to predict. 


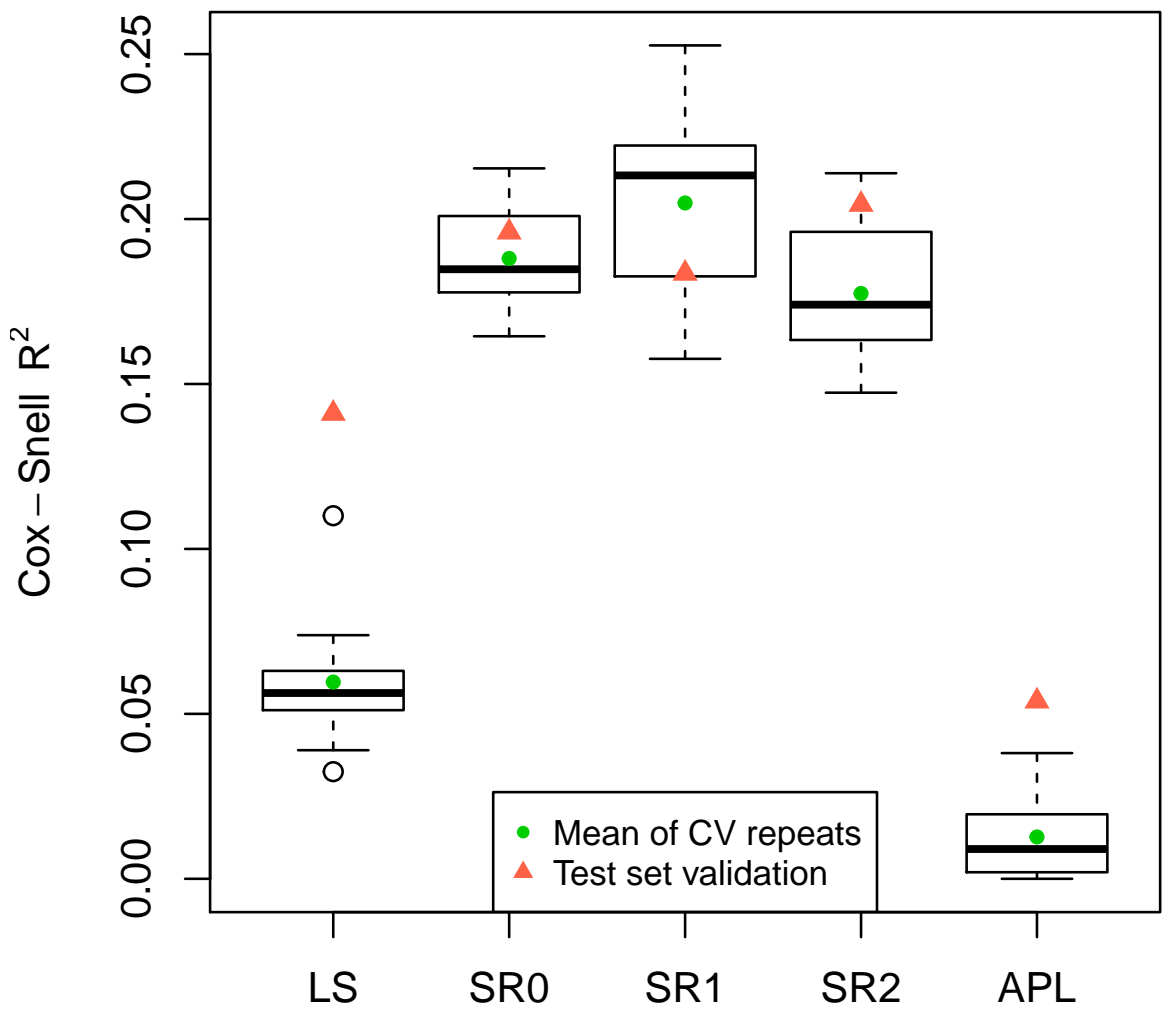

Figure 7: Maximum Cox-Snell $R^{2}$ achieved for each model. Box plots consist of the fold-averaged estimate for each of 10 repeats, so the spread of these results are due differences in the fold assignments related to the random number generation seed, and do not represent the variability in the CV estimate itself. 
LS

+ risk=Low + risk=Medium + risk=High

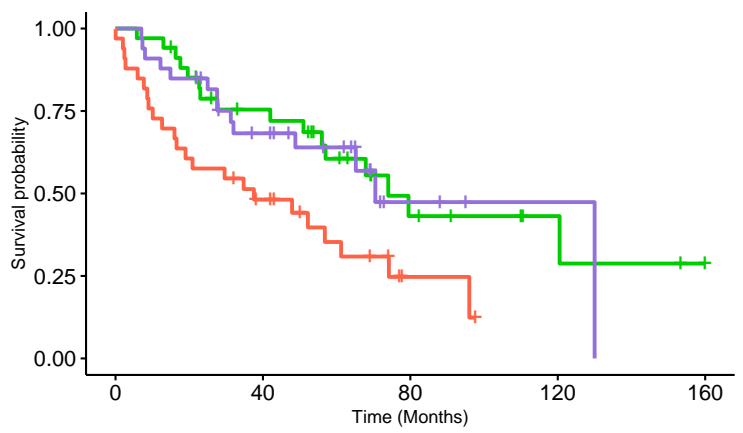

SR1

+ risk=Low + risk=Medium + risk=High

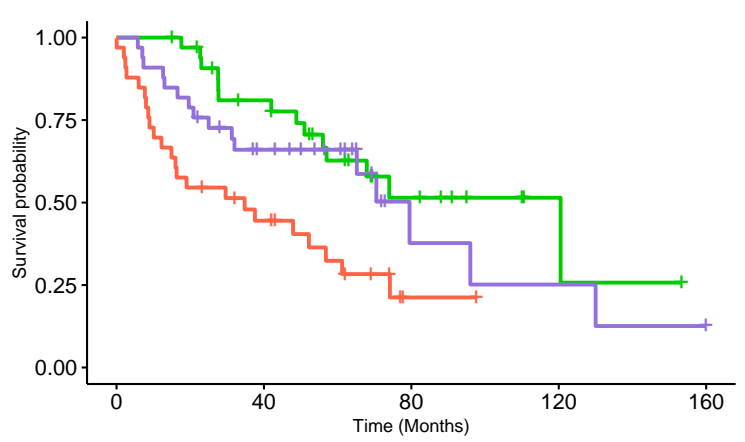

SR0

+ risk=Low + risk=Medium + risk=High

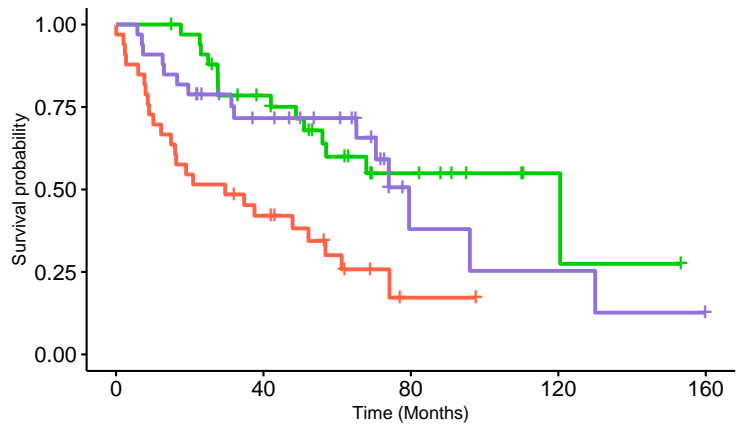

APL

+ risk=Low + risk=Medium + risk=High

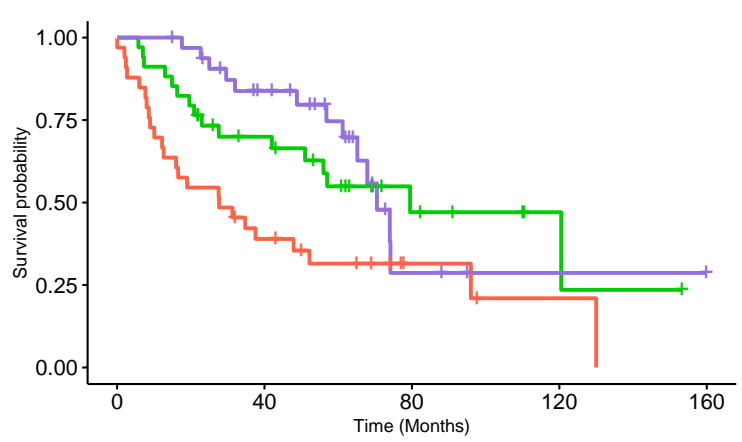

Figure 8: Risk score classification performance efficacy of each modeling framework using the test data set. More separation among stratifications on the KM plot signifies indicates that the model is doing a good job of classifying high-, medium-, and low-risk patients. LS refers to the lasso on the original covariates, SR0 refers to the SRL with proportional penalties on clinical and genetic covariates, SR1 refers to the SRL with interactions and proportional penalty weights, and APL refers to the all-pairwise lasso. 